\title{
Serious bacterial infections in neonates: improving reporting and case definitions
}

\author{
Stefania Vergnano ${ }^{a, 1, \star}$, Anna C. Seale ${ }^{b, c}$, Elizabeth J.A. Fitchett ${ }^{b}$, Mike Sharland ${ }^{a}$ and Paul T. Heath $^{a}$ \\ ${ }^{a}$ Paediatric Infectious Disease Research Group, Department of Infection and Immunity, St George's University of London, Jenner wing, \\ London, UK; ' ${ }^{b}$ MARCH Centre, London School of Hygiene \& Tropical Medicine, London, UK; 'The Farr Institute of Health Informatics \\ Research, University College London, London, UK \\ ${ }^{1}$ Present Address: Bristol Royal Hospital For Children, Department of Paediatric Infectious Diseases, Bristol, UK \\ ${ }^{*}$ Corresponding author: Tel: +44 (0) 777078 8880; E-mail: stev@doctors.org.uk
}

\begin{abstract}
Received 11 November 2016; revised 29 March 2017; editorial decision 30 March 2017; accepted 21 April 2017
Neonatal infections affect about 7 million neonates causing over 600000 deaths every year. Estimating the burden is challenging as there are multiple reporting criteria and definitions for serious bacterial infections in neonates. Essential criteria for reporting serious neonatal bacterial infections have recently been published as the STROBE-NI checklist and, in the context of maternal vaccination, definitions have been published by the Brighton Collaboration Global Alignment of Immunization safety Assessment in pregnancy (GAIA) project. Standardisation of reporting criteria is essential to allow data comparability. This an important step in providing a clearer picture of the burden of serious bacterial infections in neonates and a welcome progress for guiding new investments in interventions.
\end{abstract}

Keywords: Bacteremia, Bacterial infection, Infant, Neonatal infections, Newborn, Septicemia

In the neonatal period over 600000 deaths every year are directly due to infections, with the highest burden in low and middle income countries (LMICs). ${ }^{1}$ The burden of clinically defined possible serious bacterial infections in neonates is high. Meta-analyses of studies from sub Saharan Africa, South Asia and Latin America estimate that 6.9 million neonates need treatment for possible serious bacterial infections each year: ${ }^{2}$ this is over 3 times the yearly burden of new HIV infections in children under 15 years of age. $^{3}$ Neonatal infections and mortality are highest in the first 24 hours after birth and are disproportionally more common in low income countries, where most births occur at home. In these settings capturing the burden of early infections and deaths is logistically challenging, even as part of research studies, because births and deaths are regularly not reported.

Global estimates that specifically reflect aetiology, antimicrobial resistance patterns and outcome have never been possible due limited access to diagnosis and treatment in the highest burden settings, as well as inconsistency of clinical diagnosis. Few countries have adequate surveillance systems for neonatal infection and the burden of impairment after neonatal infection is also not well established. ${ }^{4}$

Definitions of serious bacterial infection in neonates vary and there are no universally accepted standards. Underlying this are the challenges of diagnosing serious bacterial infection, which is usually based on simple clinical algorithms due to limited laboratory facilities in LMICs. Even where available, blood cultures are often negative due to small volume samples and frequent contamination. Molecular methods using nucleic-acid extraction are highly sensitive, but bring their own challenges of interpretation and are mostly limited to research settings.

Tables 1,2 and 3 illustrate the plethora of definitions and reporting criteria used by current neonatal networks and epidemiological units, mostly based in high income countries. Substantial differences in definitions of serious bacterial infections make comparisons between studies difficult, even when raw data are compared, because different indicators are collected. Some definitions and criteria are widely used to inform research studies, but are often adapted to local circumstances rendering the resulting data incomparable.

The variation in clinical definitions and research reporting criteria for neonatal infections is partly due to the existence of a number of types of surveillance networks, which have been designed for different purposes. These include clinical networks monitoring all aspects of neonatal care (e.g. International Neonatal Network, iNEO), networks which focus on infections in (mostly adult) intensive care settings and epidemiological networks such as those coordinated by the US Centers for Disease Control (CDC) and the European Medicine Agency (EMA), which supervises the development and evaluation of new and existing drugs. There are also a limited number of surveillance groups focusing specifically on 
Table 1. Comparison of definitions for culture proven blood stream infections

\begin{tabular}{|c|c|c|c|c|c|c|c|}
\hline $\begin{array}{l}\text { Current network } \\
\text { definitions }\end{array}$ & $\begin{array}{l}\text { NEOKISS; European Centre for } \\
\text { Disease Control (ECDC) }\end{array}$ & $\begin{array}{l}\text { European Medicine } \\
\text { Agency (EMA) }\end{array}$ & $\begin{array}{l}\text { Centers for Disease } \\
\text { Control and } \\
\text { Prevention/National } \\
\text { healthcare safety } \\
\text { network (CDC-NHSN); } \\
\text { Canadian Neonatal } \\
\text { Network (CNN); } \\
\text { International } \\
\text { Nosocomial } \\
\text { Infection Control } \\
\text { Consortium (INICC) }\end{array}$ & $\begin{array}{l}\text { Egyptian Neonatal } \\
\text { Network (EGNN) }\end{array}$ & $\begin{array}{l}\text { Australian and } \\
\text { New Zealand } \\
\text { Neonatal } \\
\text { Network } \\
\text { (ANZNN) }\end{array}$ & $\begin{array}{l}\text { Neonatal Data } \\
\text { Analysis Unit (NDAU) }\end{array}$ & $\begin{array}{l}\text { Global Alignment of } \\
\text { Immunization safety } \\
\text { Assessment in } \\
\text { pregnancy (GAIA) - } \\
\text { Level } 1 \text { definition; } \\
\text { European Neonatal } \\
\text { Network (ENN); } \\
\text { Oxford Vermont } \\
\text { Neonatal Network } \\
\text { (OVN); Neonatal } \\
\text { Infection } \\
\text { Surveillance } \\
\text { Network (NEONIN) }\end{array}$ \\
\hline Microbiological criteria: ${ }^{a}$ & & & & 3 of the following: & & & \\
\hline $\begin{array}{l}\text { Pathogen isolated } \\
\text { from blood culture or } \\
\text { CSF AND Pathogen not } \\
\text { related to infection in } \\
\text { other site }\end{array}$ & $\checkmark$ & $\checkmark$ & $\checkmark$ & $\begin{array}{l}\quad \quad \checkmark \\
\text { OR } \\
\text { Positive CRP or } \\
\text { abnormal } \\
\text { haemogram }\end{array}$ & $\checkmark$ & $\checkmark$ & $\begin{array}{l}\quad \checkmark \\
\text { Recognized } \\
\text { pathogen identified } \\
\text { using a validated } \\
\text { method and from } \\
\text { a normally sterile } \\
\text { site }\end{array}$ \\
\hline $\begin{array}{l}\text { Number of additional } \\
\text { criteria: }\end{array}$ & AND at least two of: & AND at least two of: & At least one of: & $\begin{array}{l}\text { AND two or more } \\
\text { clinical signs of } \\
\text { sepsis (not } \\
\text { specified) }\end{array}$ & AND & AND at least three of: & \\
\hline $\begin{array}{l}\text { Patients receiving } \\
\text { antibiotics for at least } \\
5 \text { days (or }<5 \text { days if } \\
\text { transferred or died } \\
\text { before completion of } \\
\text { these } 5 \text { days) }\end{array}$ & & & & $\checkmark$ & $\checkmark$ & & \\
\hline Clinical signs: & & & & $\begin{array}{l}\text { Clinical picture } \\
\text { consistent with } \\
\text { neonatal infection }\end{array}$ & & & \\
\hline Temperature instability & $\begin{array}{l}\text { Fever or } \\
\text { hypothermia or } \\
\text { temperature } \\
\text { instability. }\end{array}$ & $\checkmark$ & $\checkmark$ & & & $\checkmark$ & \\
\hline Cardiovascular signs & $\begin{array}{l}\text { Tachycardia or new } \\
\text { or more frequent bradycardia. }\end{array}$ & $\checkmark$ & Bradycardia & & & $\begin{array}{ll}\checkmark & \text { Hypotension } \\
\checkmark & \text { Fall in urine } \\
\text { output }\end{array}$ & \\
\hline
\end{tabular}


Table 1. Continued

\begin{tabular}{|c|c|c|c|c|c|c|c|}
\hline $\begin{array}{l}\text { Current network } \\
\text { definitions }\end{array}$ & $\begin{array}{l}\text { NEOKISS; European Centre for } \\
\text { Disease Control (ECDC) }\end{array}$ & $\begin{array}{l}\text { European Medicine } \\
\text { Agency (EMA) }\end{array}$ & $\begin{array}{l}\text { Centers for Disease } \\
\text { Control and } \\
\text { Prevention/National } \\
\text { healthcare safety } \\
\text { network (CDC-NHSN); } \\
\text { Canadian Neonatal } \\
\text { Network (CNN); } \\
\text { International } \\
\text { Nosocomial } \\
\text { Infection Control } \\
\text { Consortium (INICC) }\end{array}$ & $\begin{array}{l}\text { Egyptian Neonatal } \\
\text { Network (EGNN) }\end{array}$ & $\begin{array}{l}\text { Australian and } \\
\text { New Zealand } \\
\text { Neonatal } \\
\text { Network } \\
\text { (ANZNN) }\end{array}$ & $\begin{array}{l}\text { Neonatal Data } \\
\text { Analysis Unit (NDAU) }\end{array}$ & $\begin{array}{l}\text { Global Alignment of } \\
\text { Immunization safety } \\
\text { Assessment in } \\
\text { pregnancy (GAIA) - } \\
\text { Level } 1 \text { definition; } \\
\text { European Neonatal } \\
\text { Network (ENN); } \\
\text { Oxford Vermont } \\
\text { Neonatal Network } \\
\text { (OVN); Neonatal } \\
\text { Infection } \\
\text { Surveillance } \\
\text { Network (NEONIN) }\end{array}$ \\
\hline Respiratory distress & $\begin{array}{l}\text { Increased oxygen requirement } \\
\text { (intubation) }\end{array}$ & $\checkmark$ Tachypnoea & & & & $\begin{array}{l}\text { Increased oxygen } \\
\text { requirement or respiratory } \\
\text { support }\end{array}$ & \\
\hline Apnoea & $\checkmark$ New or more frequent apneas. & $\checkmark$ & $\checkmark$ & & & $\begin{array}{l}\text { New or more frequent } \\
\text { apnoeas or bradycardias }\end{array}$ & \\
\hline Signs of poor perfusion & $\begin{array}{ll}\checkmark & \text { CRT }>2 \mathrm{~s} \\
\checkmark & \text { Skin color (only when } \\
& \text { recapillarization time is not used) }\end{array}$ & $\begin{array}{ll}\checkmark & \text { Sclerema } \\
\checkmark & \text { Mottled skin }\end{array}$ & & & & $\begin{array}{l}\checkmark \mathrm{CRT}>2 \mathrm{~s} \text { or impaired } \\
\text { peripheral perfusion }\end{array}$ & \\
\hline Feeding intolerance & & $\checkmark$ & & & & $\checkmark$ Ileus & \\
\hline $\begin{array}{l}\text { Central nervous } \\
\text { system signs }\end{array}$ & $\checkmark \quad$ Unstable condition, apathy & $\begin{array}{l}\checkmark \quad \text { Lethargy, } \\
\text { irritability and } \\
\text { hypotonia }\end{array}$ & & & & $\checkmark$ Lethargy & \\
\hline Glucose intolerance & New hyperglycema. & $\checkmark$ & & & & $\checkmark$ Glucose intolerance & \\
\hline $\begin{array}{l}\text { Metabolic acidosis (BE } \\
\leq 10 \mathrm{mEq} / \mathrm{L} \text { ) }\end{array}$ & $\checkmark \quad$ Unexplained metabolic acidosis & $\begin{array}{l}\checkmark \begin{array}{l}\text { or lactate }>2 \\
\mathrm{mMol} / \mathrm{L}\end{array}\end{array}$ & & & & $\begin{array}{l}\text { Unexplained metabolic } \\
\text { acidosis }\end{array}$ & \\
\hline Laboratory criteria: & & AND two of: & & & & & \\
\hline $\begin{array}{l}\text { Abnormal platelet } \\
\text { count. }\end{array}$ & & $\mathrm{Plt}<1 \times 10^{14}$ & & & & & \\
\hline Abnormal WCC & & $\mathrm{I} / \mathrm{T}>0.2$ & & & & & \\
\hline $\begin{array}{l}\text { Abnormal CRP or } \\
\text { interleukin }\end{array}$ & $\begin{array}{l}\text { Increased CRP, interleukin } \\
\text { (not included in ECDC } \\
\text { definition) }\end{array}$ & $\begin{array}{ll} & \mathrm{CRP}>15 \mathrm{mg} / \mathrm{L} \\
& \text { or procalcitonin } \\
& \geq 2 \mathrm{ng} / \mathrm{ml}\end{array}$ & & & & & \\
\hline
\end{tabular}

BE: base excess; CRP: C reactive protein; CRT: capillary refill time; CSF: cerebrospinal fluid; I/T: immature to total neutrophil ratio; mEq/L: milliequivalents per litre; Plt: platelet; WCC: white cell count.

Infection networks: INICC: international nosocomial infection control consortium (international healthcare associated infection database and infection control packages): NEOKISS: German neonatal infections net-

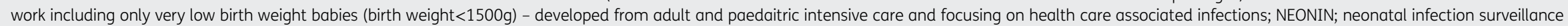
network (UK, Australia, Greece and Estonia - neonatal infection database collecting data on all neonatal infections in all infants admitted to neonatal units).

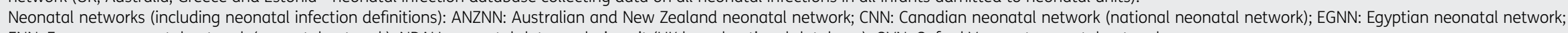
ENN: European neonatal network (neonatal network); NDAU: neonatal data analysis unit (UK based national database); OVN: Oxford Vermont neonatal network.

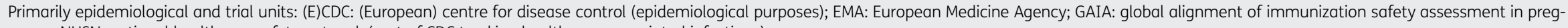
nancy; NHSN: national healthcare safety network (part of CDC tracking health care associated infections).

a EMA and ANZNN also include positive PCR/antigen testing. 
Table 2. Comparison of definitions for blood stream infections when common commensals are isolated

\begin{tabular}{|c|c|c|c|c|c|c|c|c|c|c|c|}
\hline $\begin{array}{l}\text { Bloodstream infection } \\
\text { definition criteria }\end{array}$ & \multicolumn{2}{|c|}{$\begin{array}{l}\text { NEOKISS; European Medicine } \\
\text { Agency (EMA); European Centre for } \\
\text { Disease Control (ECDC) }\end{array}$} & \multicolumn{2}{|c|}{$\begin{array}{l}\text { Centers for Disease Control } \\
\text { and Prevention/National } \\
\text { healthcare safety network } \\
(\mathrm{CDC}-\mathrm{NHSN}) \text {; Canadian } \\
\text { Neonatal Network }(\mathrm{CNN})^{\mathrm{a}}\end{array}$} & \multicolumn{2}{|c|}{$\begin{array}{l}\text { International } \\
\text { Nosocomial Infection } \\
\text { Control Consortium } \\
\text { (INICC) }\end{array}$} & \multicolumn{2}{|c|}{$\begin{array}{l}\text { European neonatal } \\
\text { network (ENN); } \\
\text { Oxford Vermont } \\
\text { neonatal network } \\
\text { (OVN) }\end{array}$} & $\begin{array}{l}\text { Neonatal Data Analysis } \\
\text { Unit (NDAU) }\end{array}$ & $\begin{array}{l}\text { Australian and New } \\
\text { Zealand Neonatal } \\
\text { Network (ANZNN) }\end{array}$ & $\begin{array}{l}\text { Global Alignment of } \\
\text { Immunization safety } \\
\text { Assessment in } \\
\text { pregnancy (GAIA) - } \\
\text { Level } 1 \text { definition }\end{array}$ \\
\hline \multicolumn{12}{|l|}{ Microbiological criteria: } \\
\hline $\begin{array}{l}\text { At least } 2 \text { or more } \\
\text { positive } B C \text { with the } \\
\text { same organism } \\
\text { drawn on separate } \\
\text { occasions (within } 2 \\
\text { days of each other) }\end{array}$ & \multicolumn{2}{|c|}{$\begin{array}{l}\text { CoNS isolated in blood culture as } \\
\text { sole pathogen }\end{array}$} & \multicolumn{2}{|r|}{$\checkmark$} & \multicolumn{2}{|r|}{$\checkmark$} & $\begin{array}{l}\text { CoNS re } \\
\text { a blood } \\
\text { from ei } \\
\text { line or } \\
\text { sample } \\
\text { from C } \\
\text { punctu } \\
\text { ventric }\end{array}$ & $\begin{array}{l}\text { overed from } \\
\text { ulture obtained } \\
\text { er a central } \\
\text { ripheral blood } \\
\text { and/or is recovered } \\
\text { obtained by lumbar } \\
\text { ventricular tap or } \\
\text { ar drain }\end{array}$ & $\begin{array}{l}\text { Mixed growth or skin } \\
\text { commensal }\end{array}$ & $\checkmark$ & $\checkmark$ \\
\hline $\begin{array}{l}\text { Number of additional } \\
\text { criteria: }\end{array}$ & \multicolumn{2}{|c|}{ AND at least two of: } & \multicolumn{2}{|c|}{ AND at least one of: } & \multicolumn{2}{|c|}{ AND at least three of: } & \multicolumn{3}{|c|}{ AND } & & \\
\hline $\begin{array}{l}\text { Patient receiving } \\
\text { antibiotics for at least } \\
5 \text { days (or }<5 \text { days if } \\
\text { transferred or died } \\
\text { before completion of } \\
\text { these } 5 \text { days) }\end{array}$ & & & & & & & \multicolumn{2}{|r|}{$\checkmark$} & & $\checkmark$ & \\
\hline \multicolumn{3}{|l|}{ Clinical signs: } & & & & & \multicolumn{2}{|c|}{ AND at least 1 of: } & & AND clinically septic & AND at least 1 of: \\
\hline $\begin{array}{l}\text { Temperature } \\
\text { instability }\end{array}$ & $\checkmark$ & $\begin{array}{l}\text { Fever or hypothermia } \\
\text { or temperature } \\
\text { instability }\end{array}$ & $\checkmark$ & $\begin{array}{l}\text { Fever, } \\
\text { hypothermia }\end{array}$ & $\checkmark$ & Fever & $\checkmark$ & $\begin{array}{l}\text { Temperature } \\
\text { instability }\end{array}$ & $\checkmark$ Temperature instability & & $\begin{array}{l}\text { Temperature } \\
\geq 37.5^{\circ} \mathrm{C} \\
\text { or }<35.5^{\circ} \mathrm{C}\end{array}$ \\
\hline Cardiovascular signs & $\checkmark$ & $\begin{array}{l}\text { Tachycardia or new } \\
\text { or more frequent } \\
\text { bradycardia }\end{array}$ & $\checkmark$ & Bradycardia & $\checkmark$ & Hypotension & $\checkmark$ & $\begin{array}{l}\text { Hemodynamic } \\
\text { instability }\end{array}$ & $\checkmark$ Hypotension & & $\begin{array}{l}\text { Tachycardia or new } \\
\text { or more frequent } \\
\text { episodes of } \\
\text { bradycardia }\end{array}$ \\
\hline $\begin{array}{l}\text { Signs of poor } \\
\text { perfusion }\end{array}$ & $\checkmark$ & $\begin{array}{l}\text { CRT>2 s or skin color } \\
\text { (only when } \\
\text { recapillarization time } \\
\text { is not used) }\end{array}$ & & & & Chills & & & $\begin{array}{l}\text { Impaired peripheral } \\
\text { perfusion (CRT }>3 \mathrm{~s} \text {, } \\
\text { pallor/mottling/ core } \\
\text { peripheral temperature } \\
\text { gap }>2^{\circ} \mathrm{C} \text {. } \\
\text { Fall in urine output }\end{array}$ & & $\begin{array}{l}\text { Pallor or poor } \\
\text { perfusion or } \\
\text { hypotension }\end{array}$ \\
\hline Apnoeas & $s$ & $\begin{array}{l}\text { New or more } \\
\text { frequent }\end{array}$ & & $\checkmark$ & & & $\checkmark$ & $\begin{array}{l}\text { OR worsening } \\
\text { respiratory } \\
\text { distress }\end{array}$ & $\begin{array}{l}\text { Clinically relevant OR } \\
\text { bradycardia episodes }\end{array}$ & & $\begin{array}{l}\text { Increased oxygen } \\
\text { requirement or } \\
\text { increased } \\
\text { requirement for } \\
\text { ventilatory support }\end{array}$ \\
\hline
\end{tabular}


Table 2. Continued

\begin{tabular}{|c|c|c|c|c|c|c|c|c|}
\hline $\begin{array}{l}\text { Bloodstream infection } \\
\text { definition criteria }\end{array}$ & \multicolumn{2}{|c|}{$\begin{array}{l}\text { NEOKISS; European Medicine } \\
\text { Agency (EMA); European Centre for } \\
\text { Disease Control (ECDC) }\end{array}$} & $\begin{array}{l}\text { Centers for Disease Control } \\
\text { and Prevention/National } \\
\text { healthcare safety network } \\
\text { (CDC-NHSN); Canadian }^{\text {Neonatal Network (CNN) }}{ }^{\text {a }}\end{array}$ & $\begin{array}{l}\text { International } \\
\text { Nosocomial Infection } \\
\text { Control Consortium } \\
\text { (INICC) }\end{array}$ & $\begin{array}{l}\text { European neonatal } \\
\text { network (ENN); } \\
\text { Oxford Vermont } \\
\text { neonatal network } \\
\text { (OVN) }\end{array}$ & $\begin{array}{l}\text { Neonatal Data Analysis } \\
\text { Unit (NDAU) }\end{array}$ & $\begin{array}{l}\text { Australian and New } \\
\text { Zealand Neonatal } \\
\text { Network (ANZNN) }\end{array}$ & $\begin{array}{l}\text { Global Alignment of } \\
\text { Immunization safety } \\
\text { Assessment in } \\
\text { pregnancy (GAIA) - } \\
\text { Level } 1 \text { definition }\end{array}$ \\
\hline Feeding intolerance & & & & & $\checkmark$ & $\checkmark$ OR Ileus & & $\begin{array}{l}\text { Difficulty in feeding } \\
\text { or abdominal } \\
\text { distention. }\end{array}$ \\
\hline $\begin{array}{l}\text { Metabolic acidosis (BE } \\
\leq 10 \mathrm{mEq} / \mathrm{L} \text { ) }\end{array}$ & & $\checkmark$ & & & & $\checkmark$ & & $\checkmark$ \\
\hline Glucose intolerance & $\checkmark$ & Hyperglycaemia & AND & & & $\checkmark$ & & \\
\hline $\begin{array}{l}\text { Other signs of } \\
\text { bloodstream } \\
\text { infection }\end{array}$ & $\checkmark$ & $\begin{array}{l}\text { Increased oxygen, } \\
\text { requirement } \\
\text { (intubation) }\end{array}$ & $\begin{array}{l}\text { Clinical signs and laboratory } \\
\text { results are not related to an } \\
\text { infection in another site }\end{array}$ & & & $\begin{array}{l}\text { Tachypnea or clinically } \\
\text { relevant increase in oxygen } \\
\text { requirement or ventilator } \\
\text { support }\end{array}$ & & \\
\hline $\begin{array}{l}\text { Central nervous } \\
\text { system signs }\end{array}$ & $\checkmark$ & $\begin{array}{l}\text { Unstable condition, } \\
\text { apathy }\end{array}$ & & & & $\begin{array}{l}\text { Lethargy, irritability or poor } \\
\text { handling }\end{array}$ & & $\begin{array}{l}\checkmark \text { Lethargy or moving } \\
\text { only when } \\
\text { stimulated or } \\
\text { hypotonia or } \\
\text { irritability }\end{array}$ \\
\hline Laboratory criteria: & AND at $\mathrm{l}$ & t one of: & & & & & & \\
\hline Abnormal CRP & $\mathrm{CRP}>2.0$ & /dl other interleukin & & & & & & $\begin{array}{l}\text { Increased number } \\
\text { of inflammatory } \\
\text { markers (CRP, } \\
\text { procalcitonin) }\end{array}$ \\
\hline Abnormal WCC & $\begin{array}{l}\text { I/T ratio } \\
\text { granuloc } \\
\text { OR leuko } \\
\text { erythrob }\end{array}$ & $\begin{array}{l}\text { (immature } \\
\text { s/total granulocytes) } \\
\text { opaenia (without } \\
\text { s) }\end{array}$ & & & & & $\checkmark$ & $\checkmark \quad$ OR I/T ratio > 0.2. \\
\hline $\begin{array}{l}\text { Abnormal platelet } \\
\text { count }\end{array}$ & Thromoc & paenia & & & & & $\checkmark$ Thromocytopaenia & $\checkmark$ \\
\hline
\end{tabular}

BC: blood cultures; BE: base excess; CoNS: coagulase negative Staphylococci; CSF: cerebrospinal fluid; CRP: C reactive protein; CRT: capillary refill time; I/T: immature to total neutrophil ratio; mEq/L: milliequivalents per litre; WCC: white cell count.

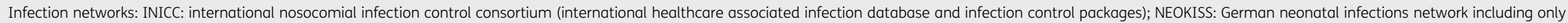
very low birth weight babies (birth weight $<1500 \mathrm{~g}$ ) - developed from adult and paedaitric intensive care and focusing on health care associated infections.

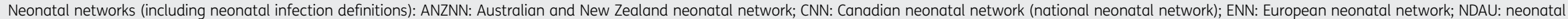
data analysis unit (UK based national database); OVN: Oxford Vermont neonatal network.

Primarily epidemiological and trial units: (E)CDC: (European) centre for disease control (epidemiological purposes); EMA: European medicine agency (providing definitions used in clinical trials); GAIA: global alignment of immunization safety assessment in pregnancy; NHSN: national healthcare safety network (part of CDC tracking health care associated infections).

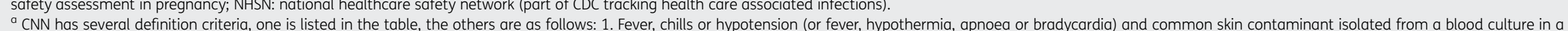

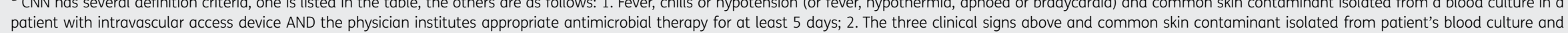
physician institutes appropriate antimicrobial therapy for at least 5 days; 3 . One of the three clinical signs above and positive antigen test on blood and organism is not related to infection in another site. 
Table 3. Comparison of definitions for culture negative blood stream infections (possible serious bacterial infection)

\begin{tabular}{|c|c|c|c|c|c|c|c|c|}
\hline $\begin{array}{l}\text { Definition for culture } \\
\text { negative blood stream } \\
\text { infection }\end{array}$ & & $\begin{array}{l}\text { SS; European } \\
\text { for Disease Control }\end{array}$ & European Medicine Agency (EMA) & $\begin{array}{l}\text { Centers for Disease } \\
\text { Control and Prevention/ } \\
\text { National Healthcare } \\
\text { Safety Network (CDC- } \\
\text { NHSN); Canadian } \\
\text { Neonatal Network (CNN) }\end{array}$ & $\begin{array}{l}\text { International } \\
\text { Nosocomial } \\
\text { Infection Control } \\
\text { Consortium } \\
\text { (INICC) }\end{array}$ & $\begin{array}{l}\text { Egyptian Neonatal } \\
\text { Network (EGNN) }\end{array}$ & 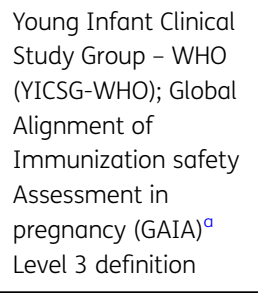 & $\begin{array}{l}\text { Global Alignment of } \\
\text { Immunization safety } \\
\text { Assessment in } \\
\text { pregnancy (GAIA) } \\
\text { Level } 2 \text { definition }\end{array}$ \\
\hline & All c & the following: & & All of the following: & $\begin{array}{l}\text { In presence of a } \\
\text { central line: }\end{array}$ & Three of the following: & $\begin{array}{l}\text { GAIA any two of the } \\
\text { following; YICSG-WHO } \\
\text { at least one of the } \\
\text { following: }\end{array}$ & \\
\hline $\begin{array}{l}\text { Antimicrobial therapy for } \\
\text { bloodstream infection } \\
\text { for at least } 5 \text { days }\end{array}$ & & $\checkmark$ & & $\checkmark$ & $\checkmark$ & $\checkmark$ & & \\
\hline $\begin{array}{l}\text { Lack of positive } \\
\text { microbiology data or no } \\
\text { organism detected }\end{array}$ & & $\checkmark$ & $\checkmark$ & $\checkmark$ & $\checkmark$ & $\begin{array}{l}\text { Positive CRP or } \\
\text { abnormal } \\
\text { haemogram }\end{array}$ & & $\checkmark$ \\
\hline $\begin{array}{l}\text { No apparent infection at } \\
\text { another site }\end{array}$ & & $\checkmark$ & $\checkmark$ & $\checkmark$ & $\checkmark$ & $\begin{array}{l}\text { Risk factors for } \\
\text { sepsis } \\
\text { (chorioamnionitis, } \\
\text { prematurity) }\end{array}$ & & \\
\hline $\begin{array}{l}\text { Number of necessary } \\
\text { criteria }\end{array}$ & ANL & t least two of: & $\begin{array}{l}\text { At least two clinical + two } \\
\text { laboratory signs: }\end{array}$ & AND at least one of: & $\begin{array}{l}\text { AND at least one } \\
\text { of: }\end{array}$ & $\begin{array}{l}\text { Two clinical signs of } \\
\text { sepsis (not specified): }\end{array}$ & & Three or more of: \\
\hline \multicolumn{9}{|l|}{ Clinical signs: } \\
\hline $\begin{array}{l}\text { Temperature } \\
\text { instability (fever or } \\
\text { hypothermia) }\end{array}$ & & $\checkmark$ & $\checkmark$ & $\checkmark$ & $\checkmark$ & & $\begin{array}{l}\geq 37.5^{\circ} \mathrm{C} \text { or } \\
<35.5^{\circ} \mathrm{C}\end{array}$ & $\begin{array}{l}\quad \text { Temperature } \\
\geq 37.5^{\circ} \mathrm{C} \text { or } \\
<35.5^{\circ} \mathrm{C}\end{array}$ \\
\hline $\begin{array}{l}\text { Signs of poor } \\
\text { perfusion }\end{array}$ & $\checkmark$ & $\begin{array}{l}\text { CRT time }>2 s \text { OR } \\
\text { skin color (only } \\
\text { when CRT is not } \\
\text { used) }\end{array}$ & & & $\begin{array}{l}\text { Hypotension } \\
\text { oliguria }\end{array}$ & & & $\begin{array}{l}\checkmark \text { Pallor or poor } \\
\text { perfusion or } \\
\text { hypotension }\end{array}$ \\
\hline Apnoea & $\checkmark$ & $\begin{array}{l}\text { New or more } \\
\text { frequent }\end{array}$ & $\checkmark$ & $\checkmark$ & & & & $\checkmark$ \\
\hline $\begin{array}{l}\text { Signs of respiratory } \\
\text { distress }\end{array}$ & $\checkmark$ & $\begin{array}{l}\text { Increased } \mathrm{O}_{2} \\
\text { requirement } \\
\text { (intubation) }\end{array}$ & $\begin{array}{l}\text { Tachypnoea (mean respiratory } \\
\text { rate }(\mathrm{RR})>2 \text { SD above normal } \\
\text { for age) or increased oxygen } \\
\text { requirements or requirement } \\
\text { for ventilation support }\end{array}$ & & & & $\begin{array}{ll}\checkmark & \mathrm{RR} \geq 60 . \\
& \text { Severe chest } \\
\text { indrawing. } \\
\text { Cyanosis (GAIA } \\
\text { only) }\end{array}$ & $\begin{array}{l}\text { Increased oxygen } \\
\text { requirement or } \\
\text { increased } \\
\text { requirement for } \\
\text { ventilatory support }\end{array}$ \\
\hline Poor feeding & & & $\begin{array}{l}\text { Feeding intolerance, poor } \\
\text { sucking abdominal distention }\end{array}$ & & & & $\begin{array}{ll}\checkmark & \text { History of } \\
\text { difficulty feeding }\end{array}$ & $\begin{array}{l}\text { Difficulty in } \\
\text { feeding or } \\
\text { abdominal } \\
\text { distention }\end{array}$ \\
\hline
\end{tabular}


Table 3. Continued

\begin{tabular}{|c|c|c|c|c|c|c|c|c|}
\hline $\begin{array}{l}\text { Definition for culture } \\
\text { negative blood stream } \\
\text { infection }\end{array}$ & $\begin{array}{l}\text { NEC } \\
\text { Cen } \\
\text { (EC }\end{array}$ & $\begin{array}{l}\text { KISS; European } \\
\text { re for Disease Control } \\
\text { C) }\end{array}$ & European Medicine Agency (EMA) & $\begin{array}{l}\text { Centers for Disease } \\
\text { Control and Prevention/ } \\
\text { National Healthcare } \\
\text { Safety Network (CDC- } \\
\text { NHSN); Canadian } \\
\text { Neonatal Network (CNN) }\end{array}$ & $\begin{array}{l}\text { International } \\
\text { Nosocomial } \\
\text { Infection Control } \\
\text { Consortium } \\
\text { (INICC) }\end{array}$ & $\begin{array}{l}\text { Egyptian Neonatal } \\
\text { Network (EGNN) }\end{array}$ & $\begin{array}{l}\text { Young Infant Clinical } \\
\text { Study Group - WHO } \\
\text { (YICSG-WHO); Global } \text { Alignment of } \\
\text { Immunization safety } \\
\text { Assessment in } \\
\text { pregnancy (GAIA) } \\
\text { Level } 3 \text { definition }^{a}\end{array}$ & $\begin{array}{l}\text { Global Alignment of } \\
\text { Immunization safety } \\
\text { Assessment in } \\
\text { pregnancy (GAIA) } \\
\text { Level } 2 \text { definition }\end{array}$ \\
\hline $\begin{array}{l}\text { Central nervous } \\
\text { system signs }\end{array}$ & $\checkmark$ & $\begin{array}{l}\text { Unstable condition, } \\
\text { apathy }\end{array}$ & $\begin{array}{l}\text { Irritability, lethargy and } \\
\text { hypotonia }\end{array}$ & & & & $\begin{array}{l}\text { Movement only } \\
\text { when } \\
\text { stimulated. } \\
\text { History of } \\
\text { convulsions }\end{array}$ & $\begin{array}{l}\text { Lethargy or } \\
\text { moving only when } \\
\text { stimulated or } \\
\text { hypotonia or } \\
\text { irritability }\end{array}$ \\
\hline $\begin{array}{l}\text { Cardiovascular } \\
\text { instability }\end{array}$ & $\checkmark$ & $\begin{array}{l}\text { Tachycardia (>200/ } \\
\text { min) or new/more } \\
\text { frequent } \\
\text { bradycardia } \\
\text { (<80/min) }\end{array}$ & $\begin{array}{l}\text { Bradycardia or tachycardia and/ } \\
\text { or unexplained persistent } \\
\text { depression over a } 0.5 \mathrm{~h} \text { time } \\
\text { period) or rhythm instability }\end{array}$ & $\checkmark \quad$ Bradycardia & & & & $\begin{array}{l}\text { Tachycardia or } \\
\text { new or more } \\
\text { frequent episodes } \\
\text { of bradycardia }\end{array}$ \\
\hline
\end{tabular}

Other signs of Petechial rash, sclerema
infections

infections

\begin{tabular}{|c|c|c|c|c|c|}
\hline Glucose intolerance & $\checkmark$ & Hyperglycaemia & $\begin{array}{l}\text { Hyperglycaemia detected at } \\
\text { least } 2 \text { times or hypoglycaemia }\end{array}$ & & \\
\hline $\begin{array}{l}\text { Unexplained } \\
\text { metabolic acidosis } \\
(\mathrm{BE} \leq 10 \mathrm{mEg} / \mathrm{l})\end{array}$ & & $\checkmark$ & $\checkmark$ OR increased serum lactate & & $\checkmark$ \\
\hline \multicolumn{6}{|l|}{ Laboratory parameters: } \\
\hline Abnormal CRP & $\checkmark$ & $\begin{array}{l}\text { Laboratory evidence } \\
\text { (CRP, interleukin) }\end{array}$ & $\checkmark$ Increased CRP or procalcitonin & $\checkmark$ & $\begin{array}{l}\text { Increased } \\
\text { number of } \\
\text { inflammatory } \\
\text { markers (CRP, } \\
\text { procalcitonin) }\end{array}$ \\
\hline Abnormal WCC & & & $\begin{array}{l}>\text { Leucopoenia or leukocytosis or } \\
>2 \times 10^{13} \text { cells } / \\
\text { Immature to total neutrophil } \\
\text { ratio }(\mathrm{I} / \mathrm{T})>0.2\end{array}$ & $\checkmark$ & OR I/T ratio $>0.2$ \\
\hline Abnormal platelet & & & $\checkmark$ Thrombocytopoenia & & $\checkmark$ \\
\hline
\end{tabular}

BE: base excess; CRP: C reactive protein; CRT: capillary refill time; I/T: immature to total neutrophil ratio; mEq/L: milliequivalents per litre; RR: respiratory rate; WCC: white cell count.

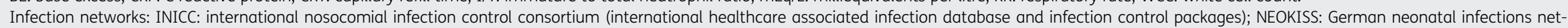
work including only very low birth weight babies (birth weight $<1500 \mathrm{~g}$ ) - developed from adult and paedaitric intensive care and focusing on health care associated infections.

Neonatal networks (including neonatal infection definitions): CNN: Canadian neonatal network (national neonatal network); EGNN: Egyptian neonatal network; YICSG-WHO: young infant clinical study group - WHO.

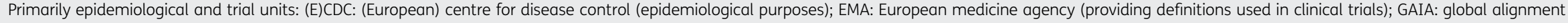
of immunization safety assessment in pregnancy; NHSN: national healthcare safety network (part of CDC tracking health care associated infections).

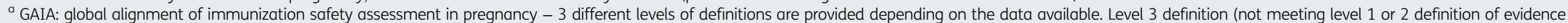
AND two or more of the following criteria): Temperature $\geq 37.5^{\circ} \mathrm{C}$ or $<35.5^{\circ} \mathrm{C}$; tachypnea or severe chest indrawing or grunting or cyanosis; change in level of activity; history of feeding difficulty; history of convulsions. 
neonatal infection (e.g. neonIN - the UK neonatal infection surveillance network). ${ }^{5}$ Such networks play a particularly important role in monitoring healthcare associated infections and are not necessarily restricted to high income countries. For example, the International Nosocomial Network Infection Control (INNIC) database is a global initiative that aims to monitor and reduce the rate of healthcare associated infections, particularly in LMICs, and includes neonates. ${ }^{6}$

Definitions used for hospitalized neonates, especially in high income countries, have limited value for early onset infection in the community or in lower level health care settings with the highest burden of disease. Simpler clinical definitions of serious bacterial infections such as the WHO young infant definition, are available and are essential for clinical decision making in low-resource settings. These have been used in some of the few available population-based studies. However, with wide variations in reporting between studies and different adaptations of the original definitions, it remains extremely challenging to reconcile differences in data for large-scale analysis of burden, aetiology and outcomes after interventions.

Addressing these issues, two recent collaborative projects have made important steps towards improved reporting of neonatal infection data. The Strengthening Publications Reporting Infections in Newborns Globally (SPRING) group was formed in 2015 with the intention of reducing some of the heterogeneity by reaching a consensus on essential reporting criteria for neonatal infection data. The group is an expert panel of clinicians, academics, researchers, epidemiologists, funders and statisticians. Following a systematic literature review, they prepared and disseminated a survey of possible reporting criteria to a large group of experts from 37 countries representing all 5 continents. The reporting criteria were then discussed in a consensus meeting and 28 final items were included in the STROBE-NI (Strengthening the Reporting of Observational Studies in Epidemiology for Neonatal Infection) checklist, published in September 2016. ${ }^{7}$ It is hoped that the STROBE-NI criteria will not only improve reporting, but also indirectly shape research and study design. Definitions for neonatal bloodstream infections, meningitis and respiratory infections have been produced by the Brighton Collaboration Global Alignment of Immunization safety Assessment in pregnancy (GAIA) project, aiming to define neonatal infection in the context of maternal vaccine studies. ${ }^{8}$

As improving newborn survival gains recognition as a key global health priority, these new international guidelines will be essential in supporting quality research in serious bacterial infections and possible serious bacterial infections, estimates of the burden of disease and for the evaluation of interventions aimed at pregnant women, neonates and infants.
Authors' contributions: SV drafted the manuscript, ACS, EJAF, MS and PTH edited and contributed to successive versions of the manuscript. SV, ACS, EJAF and PTH were involved in the SPRING group and the development of the STROBE-NI guidelines. SV and PTH coordinated the GAIA working group for the definitions of neonatal infections. SV is guarantor of the paper.

Acknowledgements: The authors thank Professor Joy Lawn for the inspiration, encouragement and support for this work.

Funding: None.

Competing interests: None declared.

Ethical approval: Not required.

\section{References}

1 Liu L, Oza S, Hogan D et al. Global, regional, and national causes of child mortality in 2000-13, with projections to inform post2015 priorities: an updated systematic analysis. Lancet 2015;385; 430-40.

2 Seale AC, Blencowe $H$, Manu AA et al. Estimates of possible severe bacterial infection in neonates in sub-Saharan Africa, south Asia, and Latin America for 2012: a systematic review and meta-analysis. Lancet Infect Dis 2014:14:731-41.

3 UNAIDS. AIDS by the Numbers. Geneva, Switzerland; UNAIDS; 2016. http://www.unaids.org/sites/default/files/media_asset/AIDS-by-the-nu mbers-2016_en.pdf [Accessed 14 October 2016].

4 Seale AC, Blencowe $\mathrm{H}$, Zaidi A et al. Neonatal severe bacterial infection impairment estimates in South Asia, sub-Saharan Africa, and Latin America for 2010. Pediatr Res 2013;74:73-85.

5 neonIN. Neonatal Infection Surveillance Network. https://www. neonin.org.uk [accessed 29 March 2017].

6 Dursun O, Ramachandran B, Villamil-Gómez W et al. Impact of a multidimensional infection control strategy on central lineassociated bloodstream infection rates in pediatric intensive care units of five developing countries: findings of the International Nosocomial Infection Control Consortium (INICC). Infection 2012; 40:415-23.

7 Fitchett EJS, Seale AC, Vergnano S et al. Strengthening the Reporting of Observational Studies in Epidemiology for Newborn Infection (STROBE-NI): an extension of the STROBE statement for neonatal infection research. Lancet Infect Dis 2016;16: e202-13.

8 Vergnano S, Buttery J, Cailes B et al. Neonatal infections: Case definition and guidelines for data collection, analysis, and presentation of immunisation safety data. Vaccine 2016; 34:6038-46. 